\title{
Implementación de actividades de aprendizaje y evaluación para el desarrollo de competencias genéricas: un caso práctico de aplicación de técnicas de Pensamiento de Diseño, y evaluación mediante rúbricas, de las competencias de Creatividad, Innovación y Emprendimiento.
}

\author{
Leonor Ruiz ${ }^{1}$, Mariluz Gordo', Marta Fernández-Diego', Andres Boza1, Llanos \\ Cuenca $^{1}$, Faustino Alarcón ${ }^{2}$ y María Del Mar Alemany-Díaz ${ }^{2}$
}

\begin{abstract}
${ }^{1}$ Escuela Técnica Superior de Ingeniería Informática. Universitat Politècnica de València (SPAIN)., lruiz@omp.upv.es, magormon@upvnet.upv.es, marferdi@omp.upv.es, aboza@omp.upv.es, llcuenca@omp.upv.es

${ }^{2}$ Escuela Técnica Superior de Ingeniería Industrial. Universitat Politècnica de València (SPAIN). faualva@omp.upv.es, mareva@omp.upv.es
\end{abstract}

\begin{abstract}
The research evidence that there are teaching practices that encourage greater student participation, resulting in a motivating work that encourages creative and innovative thinking, which enhances their autonomy and facilitate cross learning and professional skills.

Over the past year they have been incorporated techniques of design thinking, visual thinking and playful thought to the process of teaching and learning of specific contents of the subject Business Models and Functional Areas of the 4th year of Degree in Computer Engineering, with the aim of enhancing creative and innovative thinking of students. In this work the experience of educational innovation occurs.
\end{abstract}

Keywords:

Transversal skills, creativity, innovation, entrepreneurship, assessment rubrics, design thinking, methodology, evaluation

\section{Resumen}

La investigación evidencia que existen prácticas docentes que estimulan una mayor participación de los estudiantes, dando lugar a un trabajo motivador que estimula el pensamiento creativo e innovador, que potencia su autonomía y facilite el aprendizaje de competencias transversales y profesionales. 
Implementación de actividades de aprendizaje y evaluación para el desarrollo de competencias genéricas: un caso práctico de aplicación de técnicas de Pensamiento de Diseño, y evaluación mediante rúbricas, de las competencias de Creatividad, Innovación y Emprendimiento

Durante los últimos curso se han incorporado técnicas de pensamiento de diseño, pensamiento visual y pensamiento lúdico al proceso de enseñanza-aprendizaje de determinados contenidos de la asignatura Modelos de Negocio y Areas Funcionales de $4^{\circ}$ curso de Grado en Ingenieria Informática, con el objetivo de potenciar el pensamiento creativo e innovador de los estudiantes. En este trabajo se presenta dicha experiencia de innovación educativa.

Palabras clave: Competencias transversales, creatividad, innovación, emprendimiento, rúbricas de evaluación, pensamiento de diseño, metodología, evaluación

\section{Introducción}

\subsection{Concepto de “design thinking" como herramienta para resolver problemas complejos}

El Design Thinking (DT) es una aproximación metodológica a la resolución de retos y problemas de forma creativa. A través del DT se pueden investigar problemas que no están claramente definidos, obteniendo información, analizando contenido y proponiendo soluciones en los campos del diseño y la planificación. La metodología parte de un pensamiento divergente y objetivos difusos, tratando de buscar soluciones prácticas, conseguidas a través de un pensamiento convergente. Se basa en los principios de empatía, imaginación, experimentación, prototipado colectivo, pensamiento integrador y aprendizaje iterativo. Estos principios integran al usuario dentro del proceso al hacerlo partícipe tanto teórica como materialmente de la acción innovadora. Así el ser humano es el origen de la innovación puesto que se parte del análisis de sus necesidades y, mediante un proceso imaginativo e integrador, se llega a propuestas revisables para una mejora continua de los resultados.

Hoy en día el diseño está en todas partes. Su importancia desde el punto de vista funcional y estético es indudable, pero el verdadero poder del diseño radica en su dimensión estratégica, pasando a ser en estos días una clave necesaria para enfrentarnos a una realidad mucho más compleja.

La primera persona que conceptualizó y masificó el Design Thinking aplicado a los negocios fue Tim Brown (2008), profesor de la escuela de Ingeniería de Stanford University y CEO y presidente de IDEO, en un artículo publicado por Harvard Business Review . Según Tim Brown el Design thinking "es una disciplina que usa la sensibilidad y métodos de los diseñadores para hacer coincidir las necesidades de las personas, con lo 
que es tecnológicamente factible y con lo que una estrategia viable de negocios, puede convertir en valor para el cliente y en una oportunidad para el mercado"

Esto significa contemplar la innovación como un enfoque holístico donde personas, tecnología y negocio convergen en lo que se conoce como diseño. Puede ser aplicado tanto a productos y servicios, como a procesos o modelos de negocio.

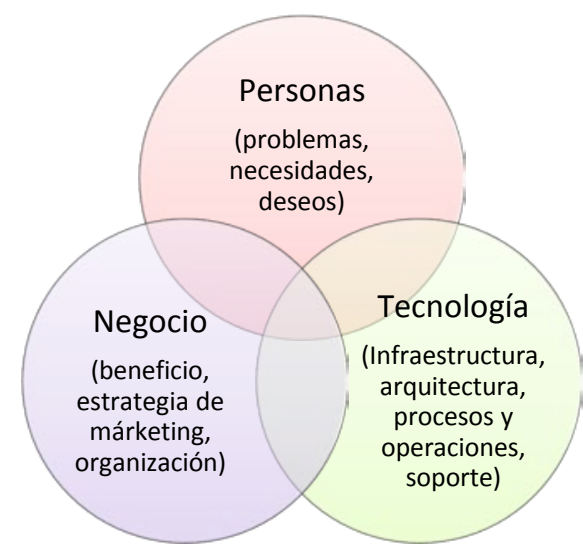

Figura 1. Enfoque holístico de la innovación

Esta visión holística se basa en encontrar la solución más innovadora teniendo en cuenta:

- $\quad$ Las necesidades, problemas y deseos de los usuarios o clientes.

- $\quad$ El papel que juega la tecnología en el diseño de la solución innovadora.

- Cómo impacta en términos cuantitativos la innovación en el negocio.

Se trata de un proceso creativo y colaborativo que permite conseguir soluciones mucho más innovadoras que las que conseguiríamos siguiendo un proceso convencional, lógico y racional.

Desde esta conceptualización, se entiende el diseño no como un mero accesorio del producto sino como un camino hacía nuevos desarrollos centrados en las necesidades reales de las personas a las que se dirigen la búsqueda de esas mismas soluciones.

Esto se debe a que el verdadero sentido del Diseño no son los objetos, sino las personas. Todo gira en torno a su realidad, a sus estilos de vida, inquietudes, necesidades, para precisamente poder crear soluciones que les ayuden a mejorar su calidad de vida.

\section{(c)) EY-NC-ND 2015, Universitat Politècnica de València}


Implementación de actividades de aprendizaje y evaluación para el desarrollo de competencias genéricas: un caso práctico de aplicación de técnicas de Pensamiento de Diseño, y evaluación mediante rúbricas, de las competencias de Creatividad, Innovación y Emprendimiento

A través de una serie de actitudes y habilidades propias de esta disciplina como la empatía, la creatividad, la colaboración, la co-creación, la visualización, la experimentación y el aprendizaje iterativo, capacita para vivir su potencial en primera persona

\subsection{El proceso de diseño}

En términos generales los procesos de diseño están compuestos por una fase divergente y otra convergente. La primera se hace preguntas, plantea, observa, identifica los problemas y encuentra inspiración para la creación de alternativas de solución. La segunda, sintetiza los hallazgos, evalúa, desarrolla, prueba y ejecuta una solución definitiva.

Design Thinking sigue un proceso que se divide en seis pasos, y que utiliza distintas herramientas para poder llevar a cabo cada uno de estos pasos.

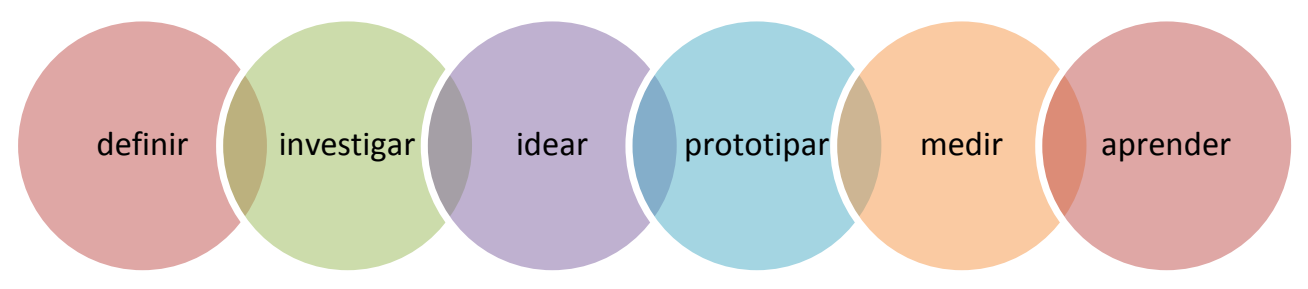

Figura 2. Proceso de Diseño

\subsubsection{Definir (y entender)}

Es el paso en el que se define formalmente el problema y se busca el vocabulario para hacerlo. Partiendo de los tres planos del enfoque holístico se pueden plantear las siguientes preguntas:

Personas: ¿Quiénes están implicados en el proceso? Definimos los actores principales (empleados, clientes, socios, usuarios).

Tecnología: ¿Qué papel juega? Definimos las implicaciones tecnológicas (montar una tienda online, rediseñar la web, utilizar otra tecnología).

Negocio: ¿Qué impacto tiene? Definimos si esta innovación genera directamente ingresos, reduce costes, mejora el margen de rentabilidad

Es por tanto un enfoque centrado en la actividad, no en el producto. El objetivo es descubrir oportunidades más allá del producto.

(cc)) EY-NC-ND 2015, Universitat Politècnica de València

Congreso IN-RED (2015) 


\subsubsection{Investigar (y observar)}

En esta etapa se busca empatizar con las distintas personas implicadas en el proceso de innovación. Esta fase se corresponde con el "Customer Discovery" de la metodología de desarrollo de clientes creada por Steve Blank (2012), y en ella se utilizan herramientas como el mapa de empatía ${ }^{1}$, y el lienzo de propuesta-valor.

Se trata por tanto de adquirir empatía con los usuarios, escuchándolos y observándolos.

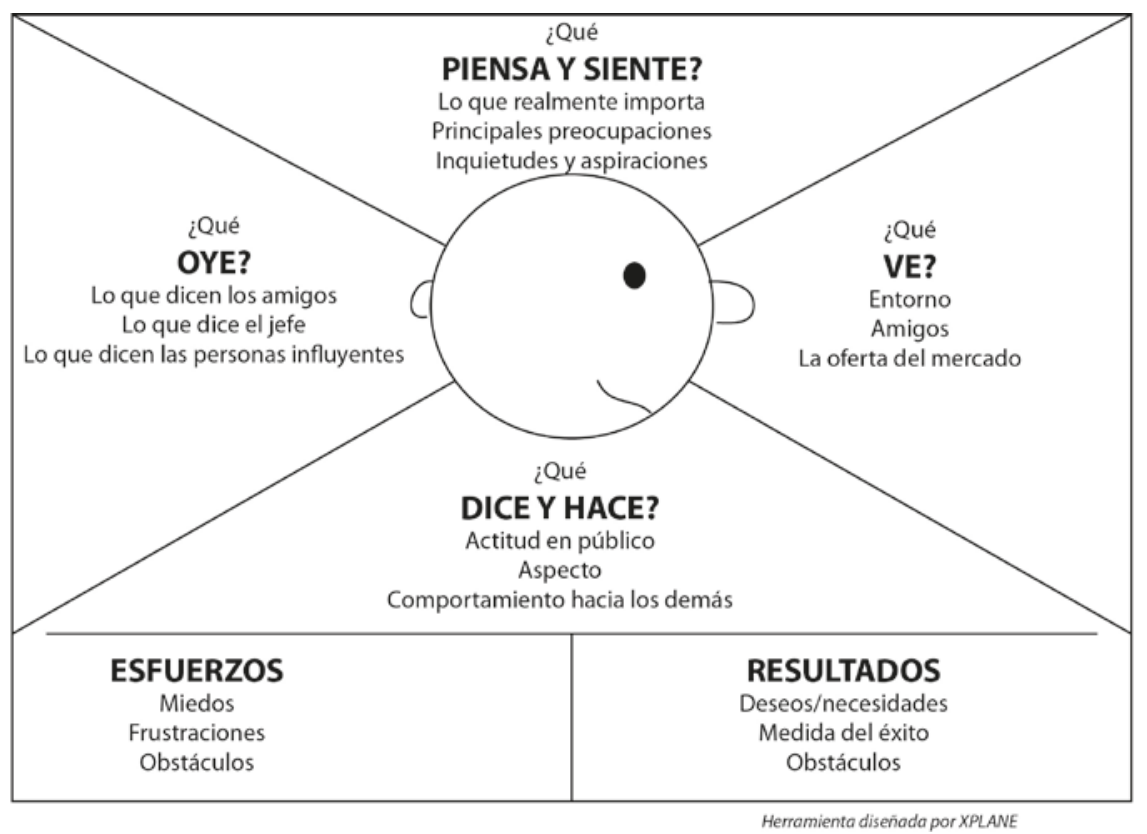

${ }^{1}$ Figura 3: Mapa de empatía

\subsubsection{Idear}

Ahora se trata de generar ideas para construir posible soluciones al problema del cliente.

En este paso podemos usar distintas herramientas para generar el mayor número de ideas posibles, como el "mapa mental” si seguimos un proceso de Brainstorming, o metodologías más avanzadas como “Lego Serious Play”.

Una vez se han generado todas las ideas, se debe intentar traducir dichas ideas en un modelo de negocio. Para ello se puede utilizar utilizando el "Lean Canvas" o un "Business Model Canvas". Si lo que pretendemos es innovar en un nuevo producto o servicio, el lienzo ayudará a ver posibles modelos de negocio para traducir esa idea en una idea de negocio viable.

\section{(cc) EY-NC-ND 2015, Universitat Politècnica de València}


Implementación de actividades de aprendizaje y evaluación para el desarrollo de competencias genéricas: un caso práctico de aplicación de técnicas de Pensamiento de Diseño, y evaluación mediante rúbricas, de las competencias de Creatividad, Innovación y Emprendimiento

Si la innovación se produce a nivel de modelo de negocio, entonces el lienzo nos muestra directamente cómo impacta la idea en el modelo actual, y cuales son los modelos hacia los que nos podríamos mover.

La idea al final de este paso es tener varios lienzos de modelos de negocio, que nos muestren alternativas para implantar esa innovación de manera efectiva en la empresa o Startup.

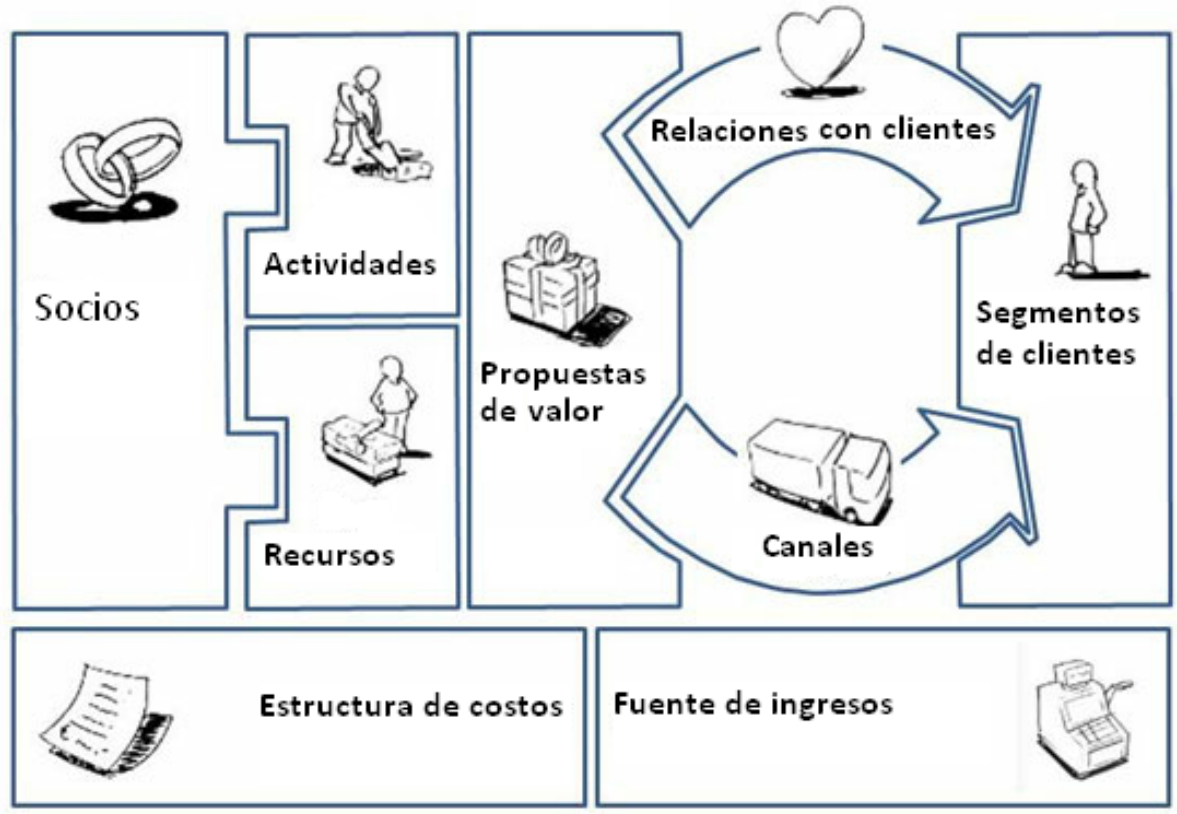

Source: http://www.businessmodelgeneration.com/ mass.pe Modelo de negocios CANVAS

Figura 4. Lienzo de Canvas (en Osterwalder y Pigneur, 2011)

\subsubsection{Prototipar}

En esta parte, siguiendo la filosofía Lean Startup (Ries 2012), se crea un producto mínimo viable (PMV). Si lo que estamos haciendo es innovar en un producto o servicio, este PMV puede ser un prototipo de dicho producto o servicio, que tenga la funcionalidad mínima requerida por el cliente. 
Leonor Ruiz, Mariluz Gordo, Marta Fernández-Diego, Andres Boza, Llanos Cuenca, Faustino Alarcón, María Del Mar Alemany-Díaz

\subsubsection{Medir}

Consiste definir las métricas relevantes para cuantificar los resultados de la innovación que pretendemos implantar en el producto, servicio o modelo de negocio.

Medir es un paso imprescindible, ya que si no podemos caer en el "innovar por innovar" y simplemente cambiar procesos, productos o modelos de negocio sin tener claro si tienen o no un impacto en la organización.

\subsubsection{Aprender}

La última etapa consiste en interpretar los resultados de la etapa anterior y ver si son satisfactorios o no. Si son satisfactorios, probablemente se pueda iterar, para diseñar un solución final del producto mínimo viable, o terminar de implantar la innovación en el modelo de negocio.

Si los resultados no son satisfactorios, entonces se puede considerar "pivorar" hacia otros modelos de negocio más rentables o diseñar nuevos prototipos.Se puede repetir el proceso "Prototipar - Medir - Aprender" varias veces teniendo en cuenta que se puede estar buscando un nuevo producto o modelo de negocio en un entorno de alta incertidumbre.

\section{Objetivos}

El objetivo de esta comunicación es mostrar la experiencia realizada para ayudar a comprender mejor algunas herramientas empleadas en la metodología del Diseño (técnicas de Design Thinking), para facilitarle la labor tanto al profesor como a los alumnos a la hora de dar soluciones creativas, y permitir desarrollar las competencias transversales de innovación, creatividad y emprendimiento, junto con la posibilidad de la evaluación de la consecución de la misma mediante rúbricas

\section{Desarrollo de la innovación}

La población de estudio fueron los estudiantes $4^{\circ}$ curso del Grado de Ingeniería Informática bajo la asignatura de la rama de Sistemas de Información: “Modelos de Negocio y Areas funcionales".

La práctica la realizaron 35 estudiantes, divididos en tres grupos oficiales de PL (prácticas de laboratorio) con edades comprendidas entre 21 y 45 años, de los cuales el $77 \%$ eran varones y el $23 \%$ mujeres.

La práctica fue organizada en una actividad de tres horas de duración del primer cuatrimestre del curso 2014-2015, La actividad que debían realizar los estudiantes fué planteada según enunciado de la tarea, publicada previamente en la intranet de la asignatura, y a realizar como práctica de laboratorio

\section{(c)) EY-NC-ND 2015, Universitat Politècnica de València}


Implementación de actividades de aprendizaje y evaluación para el desarrollo de competencias genéricas: un caso práctico de aplicación de técnicas de Pensamiento de Diseño, y evaluación mediante rúbricas, de las competencias de Creatividad, Innovación y Emprendimiento

Los estudiantes se organizaron en grupos de trabajo de 4 a 6 miembros.

Para poder llevar a cabo la práctica, se ofreció previamente información en las clases teóricas en aula sobre los conceptos de Design Thinking, Mapa de empatía, y el lienzo de Canvas, destinando para ello seis horas de clases magistrales, repartidas en cuatro sesiones.

El contenido básico de la experiencia es el siguiente:

1. Resultado/s de aprendizaje a alcanzar con la actividad:

a. Aportar sugerencias a las ideas, situaciones, casos o problemas que se plantean.

b. Utilizar técnicas de creatividad para proponer y defender ideas de calidad, originales o poco convencionales.

c. Expresar a otro las nuevas ideas que se han generado

d. Proponer ideas y soluciones innovadoras tanto en cuanto al contenido, como al proceso para llevarlas a cabo.

e. Plasmar de manera formal las ideas que se han generado.

2. Titulación, nivel y asignatura en donde se va trabajar esta competencia: $4^{\circ}$ curso Grado en Ingeniería Informática, rama Sistemas de Información. Asignatura: Modelos de Negocio y Areas Funcionales

3. Contenidos que se van a trabajar.

Pensamiento de Diseño, mapa de Empatía, modelo de negocio

4. Nombre o tipo de la actividad:

Ejercicio divergente: Primeras etapas proceso Design Thinking (definirinvestigar-idear)

5. Desarrollo:

1. Escoged y describid características de tres perfiles de clientes de un supermercado de barrio (escenificad con piezas de lego)

2. Realizad el mapa de Empatia planteando las respuestas a las diferentes preguntas basándose en el perfil de cada uno de los clientes elegidos

3. Explicar la propuesta de valor para cada uno de dichos segmento de clientes, la forma en que se relaciona con ellos y los canales utilizados para hacerles llegar las propuestas de valor, (completando así la parte derecha del lienzo Canvas).

\section{MÉTODO DE TRABAJO}

(En grupos de 4 a 6 personas)

Identificar los segmentos de mercado a los que dirigir nuestro modelo y elegir 3, escenificando con piezas de tipo Lego 
De los 3 seleccionar 1 y asignarle características (ingresos, estado civil, aficiones...)

Utilizando un mapa de Empatia y notas autoadhesivas (un color para cada miembro del equipo), responder a las siguientes preguntas:

¿qué ve en su entorno?

¿qué oye? Cómo afecta el entorno al cliente

¿qué piensa y siente en realidad?

¿qué dice y hace? Comportamiento en público y sus deseos

¿qué esfuerzos hace? Frustraciones, riesgos, qué obstáculos se interponen entre él

¿qué resultados obtiene? Qué necesita conseguir en realidad?

La creación del perfil del cliente debería permitir completar la parte derecha del lienzo de Canvas, respondiendo a preguntas como:

¿mi propuesta de valor soluciona algún problema real del cliente?

¿el cliente estará realmente dispuesto a pagar por esto?

¿cómo prefiere que se establezca la comunicación?

\section{Material:}
a. Piezas de juegos de construcción y figuras tipo Lego
b. Notas autoadhesivas de diversos colores
c. Mapa de empatía en una cartulina grande
d. Lienzo de Canvas según plantilla obtenida en la web

\section{Tiempo:}

8. Evaluación

\section{3 horas}

a. (grupo)

Se sube a PoliformaT (tareas) la memoria de la práctica que contiene

- resultado de los cuatros bloques derecha del lienzo de canvas

- fotografías de las figuras que representan los prototipos de clientes y del mapa de empatía

b. (individual)

Se entrega el test de valoración personal de la práctica

c. (profesor)

- Dispone de un diario donde se anotaron observaciones durante

el desarrollo de la sesión

- Aplicación de las Rúbricas

\section{Técnicas utilizadas:}

\section{Analisis de clientes}

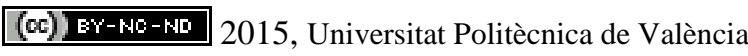


Implementación de actividades de aprendizaje y evaluación para el desarrollo de competencias genéricas: un caso práctico de aplicación de técnicas de Pensamiento de Diseño, y evaluación mediante rúbricas, de las competencias de Creatividad, Innovación y Emprendimiento

- El éxito de la innovación se basa en una profunda comprensión de los clientes, su entorno, sus rutinas diarias, sus preocupaciones y sus aspiraciones.

- Muchos modelos de negocio innovadores han triunfado porque satisfacen necesidades desatendidas de clientes nuevos o sin explotar

\section{Ideación}

- Para diseñar un modelo de negocio nuevo o innovador, hace falta un proceso creativo para generar un número elevado de ideas de modelo de negocio e identificar las mejores..

- $\quad$ La innovación en modelos de negocio no mira al pasado.

- No consiste en observar a la competencia para copiarla, sino en crear nuevos mecanismos que permitan crear valor y percibir ingresos.

- Consiste en desafiar las normas para diseñar modelos originales que satisfagan las necesidades desatendidas, nuevas u ocultas de los clientes.

- $\quad$ En la ideación hay 2 fases

- $\quad$ Generación de ideas (lo importante es la cantidad)

- Síntesis. Las ideas se comentan y combinan para escoger un número reducido de opciones viables.

\section{Pensamiento visual}

- Los esquemas ayudan a describir, comentar y comprender mejor los problemas, sobre todo los más complejos.

- Pensamiento visual es el uso de herramientas visuales como fotografías, esquemas, diagramas y notas autoadhesivas para crear significado o establecer un debate al respecto

- Notas autoadhesivas

- $\quad$ Las notas se pueden pegar, quitar y cambiar de un módulo a otro con facilidad.

- El debate que surge al final del modelo de negocio es tan importante como el resultado.

- La discusión sobre las notas que se deben colocar o quitar del lienzo, o sobre cómo un elemento afecta a los demás proporciona a los participantes un conocimiento exhaustivo del modelo de negocio y su dinámica.

- Dibujos y figuras

- Las imágenes envían un mensaje instantáneo

- Un sencillo dibujo o una figura o composición en 3D puede expresar ideas cuya explicación requeriría muchas palabras 


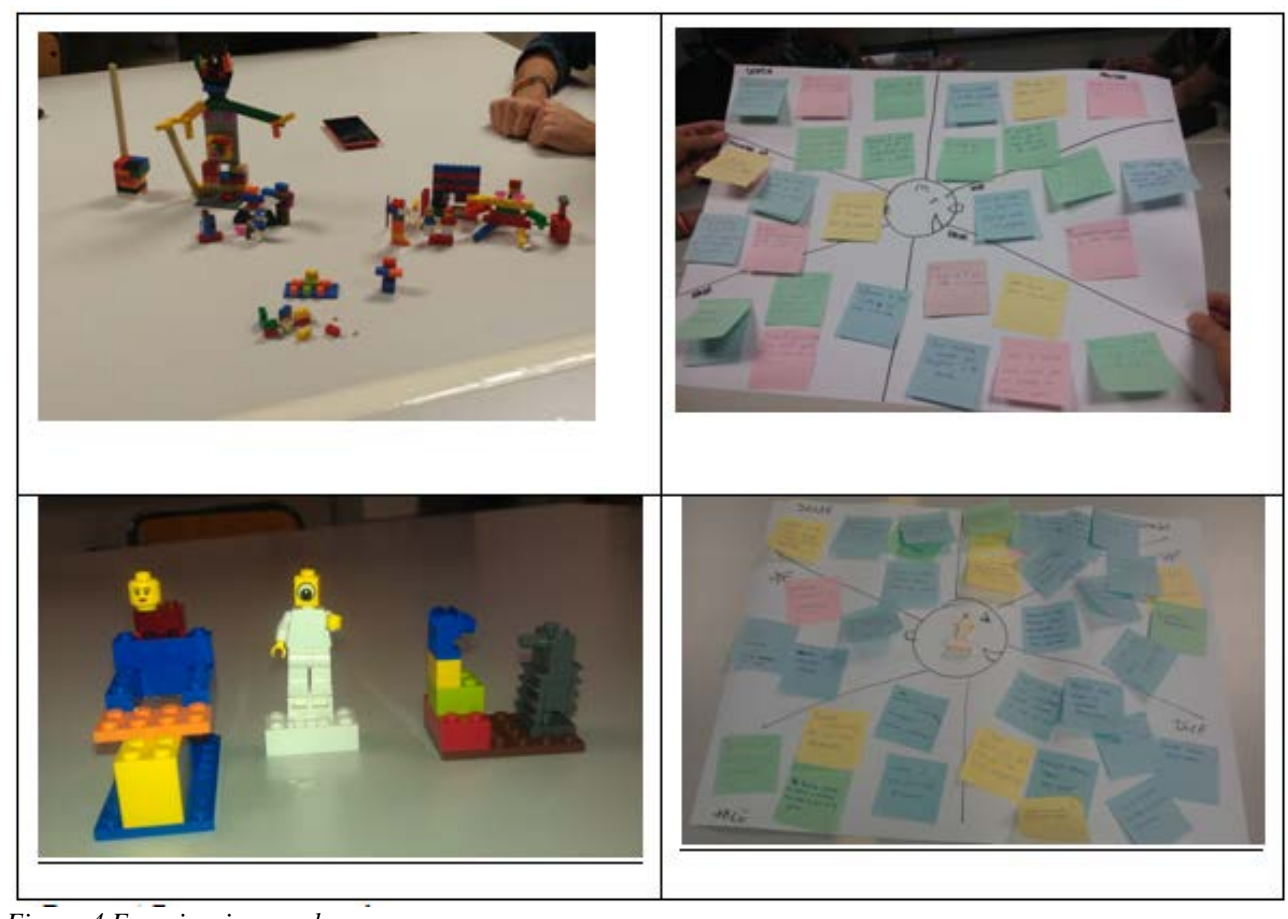

Figüra 4 Expēriencia en aula

\section{Resultados}

Las técnicas utilizadas permiten la evaluación de las competencias de creatividad, innovación y emprendimiento mediante una serie de rúbricas, así como obtener evidencias de la consecución de dichas competencias, mediantes las memorias de los trabajos realizados, fotografias e imágenes de los resultados obtenidos, dado que la singularidad de las técnicas utilizadas lo permiten.

Para evaluar esta experiencia hemos utilizado diferentes instrumentos: diario del profesor, memoria de las tareas, pos-test

Puede aplicarse para varias rúbricas sobre Creatividad e Innovación: 
Implementación de actividades de aprendizaje y evaluación para el desarrollo de competencias genéricas: un caso práctico de aplicación de técnicas de Pensamiento de Diseño, y evaluación mediante rúbricas, de las competencias de Creatividad, Innovación y Emprendimiento

\begin{tabular}{|c|c|c|c|c|c|}
\hline \multicolumn{2}{|r|}{$\begin{array}{c}\text { Perspectiva /Resultado } \\
\text { aprendizaje }\end{array}$} & Nivel 1 & Nivel 2 & Nivel 3 & Nivel 4 \\
\hline GI & $\begin{array}{l}\text { Aportar sugerencias a las } \\
\text { ideas, situaciones, casos o } \\
\text { problemas que se plantean. }\end{array}$ & $\begin{array}{l}\text { No se han aportado } \\
\text { sugerencias. }\end{array}$ & $\begin{array}{l}\text { Se han aportado } \\
\text { algunas sugerencias, } \\
\text { aplicables a casos muy } \\
\text { concretos. }\end{array}$ & $\begin{array}{l}\text { Se han aportado } \\
\text { sugerencias de } \\
\text { suficiente calidad y } \\
\text { aplicables a varios } \\
\text { casos. }\end{array}$ & $\begin{array}{l}\text { Se han aportado } \\
\text { sugerencias de buena } \\
\text { calidad y } \\
\text { ampliamente } \\
\text { aplicables. }\end{array}$ \\
\hline
\end{tabular}

Se valoran las aportaciones de cada alumno en el mapa de empatía al asignar a cada uno un color diferente en las notas autoadhesivas

\begin{tabular}{|c|c|c|c|c|c|}
\hline $\mathbf{S} / \mathbf{R}$ & $\begin{array}{l}\text { Plasmar de manera formal } \\
\text { las ideas que se han } \\
\text { generado. }\end{array}$ & $\begin{array}{l}\text { No se comprenden } \\
\text { las ideas generadas } \\
\text { ya que no se } \\
\text { expresan con } \\
\text { claridad }\end{array}$ & $\begin{array}{l}\text { Se comprenden las } \\
\text { ideas generadas pero se } \\
\text { describen de manera } \\
\text { inadecuada }\end{array}$ & $\begin{array}{l}\text { Se comprenden las ideas } \\
\text { generadas y se } \\
\text { describen de manera } \\
\text { adecuada pero poco } \\
\text { precisa }\end{array}$ & $\begin{array}{l}\text { Se comprenden las } \\
\text { ideas generadas y se } \\
\text { describen de manera } \\
\text { precisa y formal. }\end{array}$ \\
\hline & \multicolumn{5}{|c|}{ Analizar la forma de expresión y el numero de propuestas de valor en el lienzo de Canvas } \\
\hline \multirow[t]{2}{*}{ GI } & $\begin{array}{l}\text { Proponer ideas y soluciones } \\
\text { innovadoras tanto en cuanto } \\
\text { al contenido, como al } \\
\text { proceso para llevarlas a } \\
\text { cabo. }\end{array}$ & $\begin{array}{l}\text { No se proponen ni } \\
\text { ideas ni soluciones } \\
\text { innovadoras }\end{array}$ & $\begin{array}{l}\text { Se propone el contenido } \\
\text { alguna idea o solución } \\
\text { innovadora pero no se } \\
\text { especifica cómo } \\
\text { implementarlas }\end{array}$ & $\begin{array}{l}\text { Se propone el contenido } \\
\text { de diversas ideas o } \\
\text { soluciones innovadoras } \\
\text { y se esboza el } \\
\text { procedimiento para } \\
\text { llevarlas a cabo. }\end{array}$ & $\begin{array}{l}\text { Se propone el } \\
\text { contenido de diversas } \\
\text { ideas o soluciones } \\
\text { innovadoras y se } \\
\text { define de manera } \\
\text { precisa el } \\
\text { procedimiento para } \\
\text { llevarlas a cabo. }\end{array}$ \\
\hline & \multicolumn{5}{|c|}{$\begin{array}{l}\text { A través de las propuestas de valor que anotan en el lienzo de Canvas, analizar cantidad y calidad, } \\
\text { asi como los canales y los tipos de relación propuestos }\end{array}$} \\
\hline \multirow[t]{2}{*}{ CRS } & $\begin{array}{l}\text { Utilizar técnicas de } \\
\text { creatividad para proponer y } \\
\text { defender ideas de calidad, } \\
\text { originales o poco } \\
\text { convencionales. }\end{array}$ & $\begin{array}{l}\text { No se utilizan } \\
\text { técnicas de } \\
\text { creatividad para } \\
\text { analizar y } \\
\text { solucionar } \\
\text { problemas }\end{array}$ & $\begin{array}{l}\text { Se utilizan técnicas de } \\
\text { creatividad } \\
\text { ocasionalmente, menos } \\
\text { de un } 50 \% \text { de las veces. }\end{array}$ & $\begin{array}{l}\text { Se utilizan técnicas de } \\
\text { creatividad } \\
\text { frecuentemente, más de } \\
\text { un } 50 \% \text { de las veces. }\end{array}$ & $\begin{array}{l}\text { Siempre que es } \\
\text { necesario, se utilizan } \\
\text { técnicas de } \\
\text { creatividad para } \\
\text { analizar y resolver } \\
\text { problemas }\end{array}$ \\
\hline & \multicolumn{5}{|c|}{$\begin{array}{l}\text { Una vez conocen las herramientas expuestas en esta práctica, en la siguiente con otro planteamiento } \\
\text { observar y comprobar en la memoria e numero de técnicas de diseño utilizadas }\end{array}$} \\
\hline C & $\begin{array}{l}\text { Expresar a otro las nuevas } \\
\text { ideas que se han generado. }\end{array}$ & $\begin{array}{l}\text { No se aprecia } \\
\text { predisposición a } \\
\text { expresar nuevas } \\
\text { ideas }\end{array}$ & $\begin{array}{l}\text { Las ideas que se han } \\
\text { generado son } \\
\text { expresadas en algunas } \\
\text { ocasiones y en grupos } \\
\text { reducidos }\end{array}$ & $\begin{array}{l}\text { Las ideas que se han } \\
\text { generado son } \\
\text { expresadas en la } \\
\text { mayoría de las } \\
\text { ocasiones en grupos } \\
\text { más amplios }\end{array}$ & $\begin{array}{l}\text { Las ideas generadas } \\
\text { son siempre } \\
\text { expresadas hacia } \\
\text { todos los grupos }\end{array}$ \\
\hline & \multicolumn{5}{|c|}{$\begin{array}{l}\text { Mediante observación el profesor anota las intervenciones de cada alumno en el debate en grupo en } \\
\text { el brainstorming. Hay un turno de palabra para explicar la propuesta de clientes de cada uno }\end{array}$} \\
\hline
\end{tabular}

Tabla 1. Rúbrica de evaluación (Adaptado de Cuenca et al., 2015)

La valoración por parte de los alumnos, medida a través del test, fue altamente positiva en cuanto al interés, nivel de ejecución, contenidos y valoración global, tal y como aparece en la siguiente tabla (A: de acuerdo. I: indiferente, D: desacuerdo) 
Leonor Ruiz, Mariluz Gordo, Marta Fernández-Diego, Andres Boza, Llanos Cuenca, Faustino Alarcón, María Del Mar Alemany-Díaz

\begin{tabular}{|l|c|c|c|}
\hline Interes & $\mathrm{A}$ & $\mathrm{I}$ & $\mathrm{D}$ \\
\hline Considero que esta actividad ayuda en el aprendizaje de la asignatura & $74 \%$ & $26 \%$ & $0 \%$ \\
\hline Considero que es de utilidad para mi futura carrera profesional & $46 \%$ & $37 \%$ & $17 \%$ \\
\hline Me hace pensar & $60 \%$ & $29 \%$ & $11 \%$ \\
\hline Es divertida & $94 \%$ & $0 \%$ & $6 \%$ \\
\hline Es interesante & $91 \%$ & $9 \%$ & $0 \%$ \\
\hline Me hace ver otros puntos de vista & $86 \%$ & $9 \%$ & $6 \%$ \\
\hline Ejecución & $\mathrm{A}$ & $\mathrm{I}$ & $\mathrm{D}$ \\
\hline La información o documentación para su preparación ha sido adecuada & $91 \%$ & $6 \%$ & $3 \%$ \\
\hline Me ha resultado sencilla la realización de la actividad & $100 \%$ & $0 \%$ & $0 \%$ \\
\hline Las condiciones del lugar eran adecuadas & $74 \%$ & $20 \%$ & $6 \%$ \\
\hline El clima de trabajo en el aula era bueno & $100 \%$ & $0 \%$ & $0 \%$ \\
\hline Me he sentido a gusto trabajando & $100 \%$ & $0 \%$ & $0 \%$ \\
\hline Contenidos & $\mathrm{A}$ & $\mathrm{I}$ & $\mathrm{D}$ \\
\hline Esta práctica/seminario refuerza mis destrezas funcionales & $74 \%$ & $20 \%$ & $6 \%$ \\
\hline Esta práctica/seminario refuerza mis destrezas técnicas & $66 \%$ & $26 \%$ & $9 \%$ \\
\hline Refuerza mi capacidad de comunicación & $89 \%$ & $9 \%$ & $3 \%$ \\
\hline Refuerza mi capacidad de trabajo en equipo & $91 \%$ & $6 \%$ & $3 \%$ \\
\hline Valoración global & $\mathrm{A}$ & $\mathrm{I}$ & $\mathrm{D}$ \\
\hline Considero la actividad muy interesante & $77 \%$ & $17 \%$ & $6 \%$ \\
\hline Estoy satisfecho con el trabajo realizado & $86 \%$ & $11 \%$ & $3 \%$ \\
\hline Ha aumentado mi interés por los temas tratados y por la asignatura & $14 \%$ & $6 \%$ \\
\hline Tabla 2 Encuesta de valoración de los alumnos & & \\
\hline
\end{tabular}

\section{Conclusiones}

En este trabajo se ha presentado una experiencia de innovación educativa siguiendo una metodología que incluye herramientas que permiten potenciar las competencias transversales de creatividad e innovación de los estudiantes en un contexto de enseñanzaaprendizaje universitario a través del pensamiento de diseño, pensamiento visual y pensamiento lúdico.

Se analizó el problema de la evaluación de competencias, que requiere una evaluación auténtica o realista y una evaluación de la ejecución o cumplimiento de las tareas, exigiendo a los estudiantes su implicación, planificación, desarrollo, comunicación y argumentación de las mismas

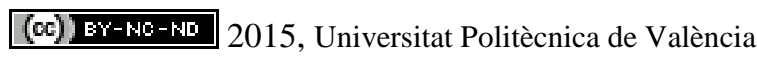

Congreso In-Red (2015) 
Implementación de actividades de aprendizaje y evaluación para el desarrollo de competencias genéricas: un caso práctico de aplicación de técnicas de Pensamiento de Diseño, y evaluación mediante rúbricas, de las competencias de Creatividad, Innovación y Emprendimiento

El trabajo con metodologías de innovación y creatividad, favorecen la implicación y motivación de los estudiantes. Además, permiten desarrollar el espíritu emprendedor de los alumnos en estas fases de formación académica

Por último destacar, que desde el punto de vista del profesorado, la experiencia desarrollada ha sido muy gratificante, ya que hemos visto un alumnado altamente motivado, con aportaciones creativas e innovadores, y una alta participación,lo que nos anima a seguir en esta línea de trabajo.

\section{Referencias}

BROWN, TIM (Junio 2008). Design Thinking ._Harvard Business Review

BROWN, TIM (Septiembre 2009): Change by Design: How Design Thinking transforms organization and inspires innovation. HarperCollinsPublishers

BROWN TIM AND WYATT, J. (2010) Design Thinking and Social Innovation Stanford Social Innovation Review.

$<$ http://www.ssireview.org/articles/entry/design_thinking_for_social_innovation/> [Consulta:12 de marzo de 2015]

CUENCA L., BOZA A., FERNÁNDEZ-DIEGO M., RUIZ L., GORDO ML., ALARCÓN F., POLER R., ALEMANY MME. Rubric to assess the competence of innovation, creativity and entrepreneurship in bachelor degree. XXI International Conference on Industrial Engineering and Operations Management Aveiro (Portugal) from July 6th-8th, 2015.

GRAY D., BROWN S., MACANUFO J. (2012). Gamestorming: 83 juegos para innovadores, inconformistas y generadores del cambio. Madrid. Deusto

GONZÁLEZ GONZÁLEZ, CARINA SOLEDAD (2014) "Estrategias para trabajar la creatividad en la Educación Superior: pensamiento de diseño, aprendizaje basado en juegos y en proyectos". en RED. Revista de Educación a Distancia, núm. 40, enero-marzo, 2014, pp. 7-22 Universidad de Murcia

OSTERWALDER, A. Y PIGNEUR, Y. (2011). Generación de Modelos de Negocio. Deusto

RIES, ERIC (2012). El método de Lean Startup. Deusto S.A. Ediciones

STEVE BLANK Y BOB DORF (2012) The Statup Owner`s Manunal. K \& S Ranch

SOULSIGHT, DESIGN THINKING. Diseño el cambio. Reto mundial de escuelas Toolkit para profesores. $<$ http://redemprendimientoinacap.cl/docentes/wp- 
Leonor Ruiz, Mariluz Gordo, Marta Fernández-Diego, Andres Boza, Llanos Cuenca, Faustino Alarcón, María Del Mar Alemany-Díaz

content/files_mf/13987217341380900046toolkit_innovacionenaula.pdf> [Consulta: 14 de febrero de 2015]

WARE, C. (2008). Visual Thinking: for Design. Burlington: Morgan Kaufmann.

\section{(c)) EY-NC-ND 2015, Universitat Politècnica de València}

Congreso In-Red (2015) 